Research Article

\title{
Dynamic Behaviors of Contact Lines on Micropillared Hydrophobic Surfaces
}

\author{
Chen-chuan Tan, Zhi-hai Jia, Hui-nan Yang, and Tai-min Cai \\ School of Energy and Power Engineering, University of Shanghai for Science and Technology, Shanghai 200093, China \\ Correspondence should be addressed to Zhi-hai Jia; zhhjia@usst.edu.cn
}

Received 10 December 2015; Revised 9 March 2016; Accepted 15 March 2016

Academic Editor: José L. Ocaña

Copyright ( $) 2016$ Chen-chuan Tan et al. This is an open access article distributed under the Creative Commons Attribution License, which permits unrestricted use, distribution, and reproduction in any medium, provided the original work is properly cited.

The dynamic characteristics of contact lines on inclined micropillared surfaces were investigated in this paper. It was observed that the contact lines varied gradually to a ladder shape with the droplet sliding on micropillared surfaces under Wenzel state. The dynamic deformation of contact lines would be more obvious under Wenzel state and Cassie impregnating state; however it is negligible when the droplet is in one-dimensional scenario. Droplet layers formed during droplet sliding were left behind and evaporated quickly and disappeared. Based on these characteristics, the comparison of experimental data with theoretical models was discussed. It was found that energy barrier played an important role in analyzing wetting characteristics. Because of ignoring the role of energy barrier, the model of sliding angle cannot predict the sliding angle on micropillared surfaces very well, especially when the area fraction is small. This work is helpful to propose a more accuracy sliding angle model.

\section{Introduction}

Micro/nanostructured surfaces have attracted much attention in both academia and industry because of their great application of drag reduction [1], anticorrosion [2-5], antiicing [6], waterproof coating [7], self-cleaning device [3, 8, 9], medicine [10], and other fields. In recent years, the size of microstructures can be controlled to nanolevel with the rapid development of nanotechnology; research of the impact of the microstructures' size and shape on wetting characteristics is of great interest.

Wetting state is not only determined by solid-liquid contact areas, but also related to wetting of three-phase contact lines [11]. Gao and McCarthy $[12,13]$ found that the advancing and receding angle were actually the results that the contact line overcomes the energy barrier. Thiele and Knobloch [14] considered two types of heterogeneity: the front-end of the film substrate was hydrophobic, while the rear-end was hydrophilic; then the stick-slip motion of droplet was studied numerically. Raj et al. [15] acquired two energy equations about variation characteristics of three-phase contact line and the wetting area when attempting to derive the contact angle hysteresis model on superhydrophobic surfaces. Snoeijer and
Andreotti [16] discussed microscopic processes that were proposed to resolve the moving contact line paradox and identify the different dynamical regimes of contact line motion.

For microstructured surfaces, the droplet started to slide as the receding contact line detached from the top of a pillar and jumped to the next pillar [17]. Based on this hypothesis, Dorrer and Rühe [18] proposed that the energy barrier was overcome by a meniscus detaching from pillars. It was found that the energy barrier was mainly a function of the wetted area between the droplet and an individual pillar, and the advancing contact angle would reach $180^{\circ}$ during the advancing motion. Widom [19] also predicted the "pouring" motion by considering the effect of line tension on droplet contact angles and adhesion characteristics [20-23].

In fact, when a Cassie droplet is moved across a surface, the advancing portion of the contact line simply lies down onto the next roughness feature and this portion contributes negligible resistance to droplet motion. The deformations due to pinning [24] are localized at the receding region, and as the contact line is displaced, it is hypothesized that microcapillary bridges $[25,26]$ are formed at each pinning site [27-29]. The amplificatory images were actually captured 


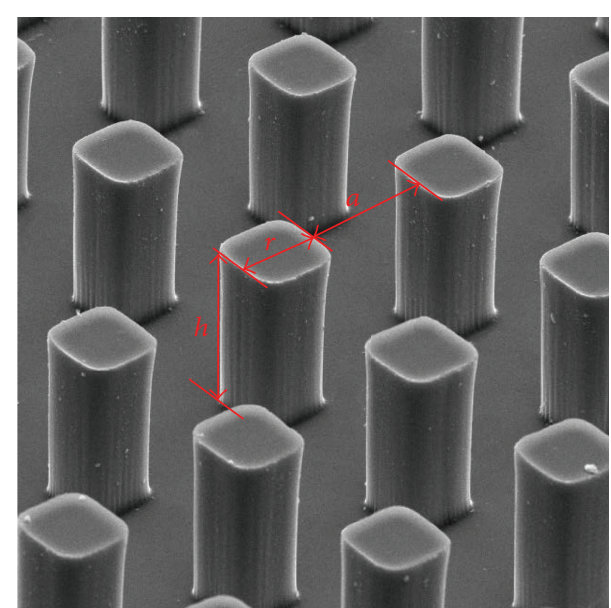

(a)

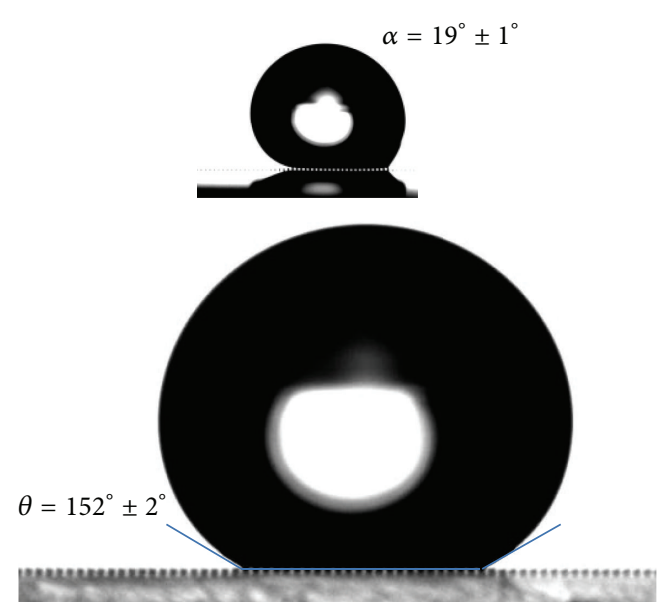

(b)

FIGURE 1: (a) SEM image ( $a$ is the pillar spacing, $h$ is the height of the pillars, and $r$ is the width) and (b) wetting properties of micropillared surfaces.

by environmental scanning electron microscope (ESEM) [30].

In recent years, all proposed relations for sliding angle of a droplet on a smooth surface were reduced into the following unified model by Wolfram et al. [31, 32]:

$$
\sin \alpha=k \frac{2 \pi r}{m g}
$$

where $m$ denotes the droplet mass quality, $g$ is the gravity acceleration, $r$ is the radius of the wetted area, and $k$ is a constant. And Miwa et al. [33] assumed that the surface had a series of uniform needles and proposed a model describing sliding angles on rough surfaces in the following form:

$\sin \alpha$

$$
=\frac{2 r f k \sin \theta^{\prime}\left(\cos \theta^{\prime}+1\right)}{g(r \cos \theta+1)}\left[\frac{3 \pi^{2}}{m \rho^{2}\left(2-3 \cos \theta^{\prime}+\cos ^{3} \theta^{\prime}\right)}\right]^{1 / 3},
$$

where $\alpha_{0}$ is the sliding angle for smooth surfaces, $V$ is the mass volume, $\theta^{\prime}$ is the equilibrium contact angle on a rough surface, and $\theta$ is the equilibrium contact angle on a flat surface. Furthermore, Lv et al. [34] derived a new sliding angle model for micropillared hydrophobic surfaces as follows:

$\sin \alpha$

$$
\begin{aligned}
& =\frac{2 \sqrt[3]{3} \gamma_{\mathrm{lv}} \sqrt{2\left(1+\cos \phi_{0}\right)-\left(1+\cos \phi_{0}\right)^{2} f}}{\rho g \sqrt[3]{\pi V^{2}} \sqrt[3]{4-3\left(1+\cos \phi_{0}\right)^{2} f^{2}+\left(1+\cos \phi_{0}\right)^{3} f^{3}}}(1 \\
& \left.+\cos \phi_{0}\right) f
\end{aligned}
$$

where $\gamma_{\mathrm{lv}}$ is the surface tension between droplet and vapor, $f$ is the area fraction of the surface, and $\phi_{0}$ is Young's contact angle. It was showed that the theoretical values are consistent with the experimental data when the area fraction is low, but not for larger area fraction.
To the author's knowledge, the dynamical characteristics of the contact line remain unclear in the previous research. Here, dynamic characteristics of contact lines under different wetting states on micropillared surfaces were investigated, and the droplet layer effects on the sliding angle were discussed, and then experimental results were compared with theoretical models.

\section{Experimental Materials and Methods}

Micropillared hydrophobic surfaces were fabricated by photolithography and etching of inductively coupled plasma (ICP), with same side lengths, but different spaces and heights, as shown in Figure 1(a). The pillars are uniformly distributed in a rectangular grid with different area fraction $f$, where $f=a^{2} /(a+r)^{2}$ and $a$ is the spacing between the pillars. The thick silicon wafer (SU8-25) was first covered with a photoresist layer by spin coating, and a lithography mask was then covered on SU8-25 to form a mold. Then polydimethylsiloxane (PDMS) was poured into a mold in a vacuum oven at $90^{\circ} \mathrm{C}$ for about 60 minutes, and the remaining PDMS polymer was removed from the silicon wafer. Finally, the surface was soaked in anhydrous alcohol for 25 minutes and then cleaned by ultrasonic cleaner for 5 minutes and then dried.

The sliding angle was related to the volume of the droplet; that is, as the droplet size increased, the sliding angle decreased. The contact angle was measured to be $113 \pm 1^{\circ}$ on the PDMS surface, and droplets cannot slide off even when the inclination angle was larger than $90^{\circ}$. The contact angle of droplet on square-pillar surface was $152 \pm 2^{\circ}$, and the sliding angle was $19 \pm 1^{\circ}$, as shown in Figure 1(b).

In this study, water droplets with different volumes were gently deposited on micropillared surfaces by a syringe. A contact angle meter was utilized to measure sliding angles. With increasing inclination angle, the droplet starts to deform. When the inclination angle increases to a critical 


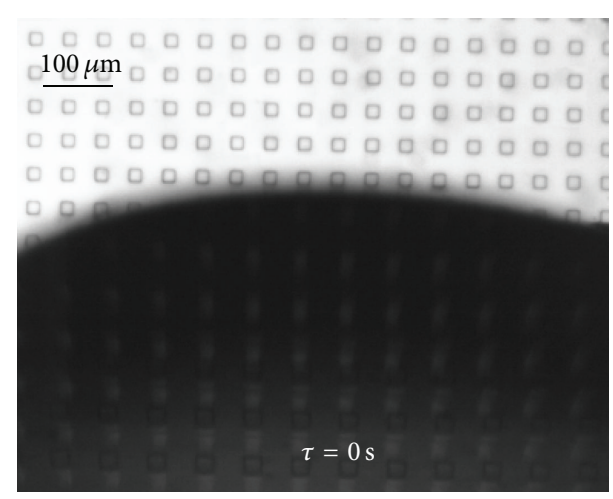

(a)

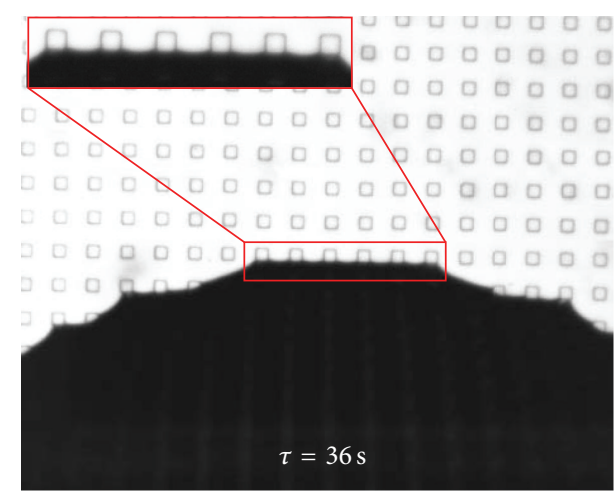

(c)

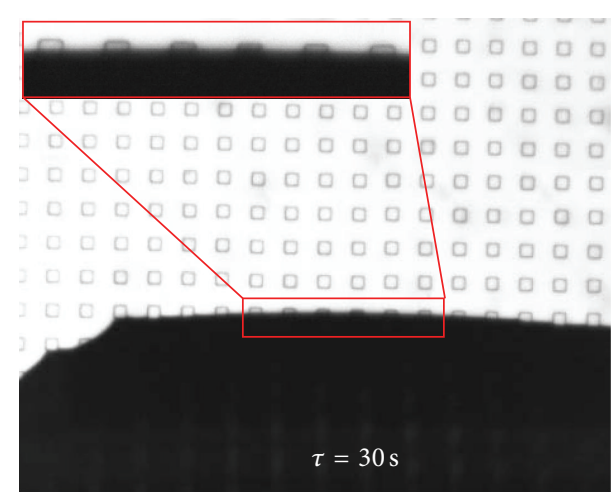

(b)

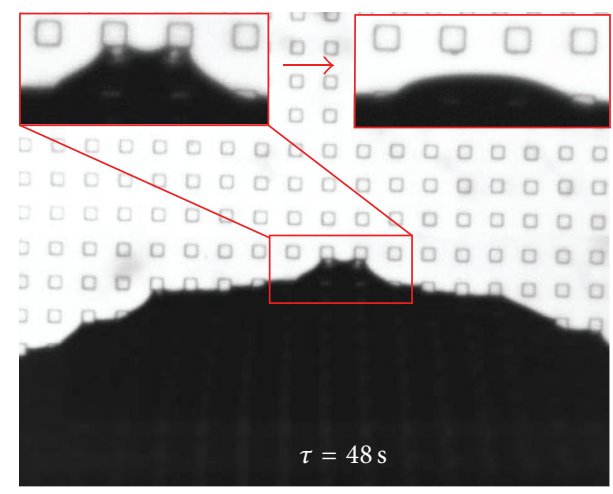

(d)

FIgURE 2: Contact line of a Wenzel state droplet on micropillared surfaces.

value, the droplet begins to slide. The instantaneous images of the droplet on the surface were recorded with a high-speed camera.

\section{Results and Discussion}

Various wetting states occur on rough surfaces; as a result, the dynamic behaviors of receding contact lines were different during droplet sliding process. Here, a Wenzel droplet was deposited gently on a micropillared surface. The images of receding contact line during the droplet moving were captured and shown in Figure 2(a). The receding contact line was about to move after $30 \mathrm{~s}$ and changed into a ladder shape gradually, as shown in Figure 2(b). However, the middle of contact line was always adhered to the pillars. After $36 \mathrm{~s}$, the droplet started to slide forward under gravity due to the increasing inclination angle; both the left and right sides of the contact line appeared to be a ladder shape obviously, but the middle of contact line was still adhered to the pillars which were enlarged in Figure 2(c). The gravity component increases with the increasing of inclination angle; the receding contact line was drawn along the direction of moving. Then the middle of contact line was detached from the pillars when the cohesive force reached a critical value and droplet layer remained on the side of pillars, as shown in Figure 2(d). Therefore, the profile of the receding contact line was obviously varied with time, so that the apparent contact angle of droplet and wetting area was constantly changing, which causes the error between the theoretical data and experimental results.

Except for the Wenzel and Cassie state, the Cassie impregnating state was also considered. The liquid penetrated into grooves between pillars and pillar "islands" ahead of the droplet were dry, as shown in Figure 3(a). Compared with the Wenzel state depicted in Figure 3(b), the contact line of the Cassie impregnating state was larger and wider (I indicated the contact line for Wenzel state; II indicated the contact line for Cassie impregnating state). During droplet sliding process, the deformation of receding contact lines would be more obvious; it was also shown in the difference between the theoretical and experimental results.

Moreover, the different dimension of droplet wetting also affected dynamic behaviors of contact lines; like Wenzel state, all spaces between pillars underneath the droplet were filled with liquid which was named two-dimensional scenario $[35,36]$. However, under external stimuli such as impact, pressure, vibration, or electrowetting, only the spaces near the contact line were filled with liquid as shown in Figure 4(a), which was named one-dimensional scenario. If the wetting state would not be transformed into the two-dimensional scenario during droplet sliding process, the ladder-shape steps were less than for Wenzel state due to the reducing of wetting area, as shown in Figure 4(b) (III indicates the boundary of liquid and air; IV indicates the contact line for 


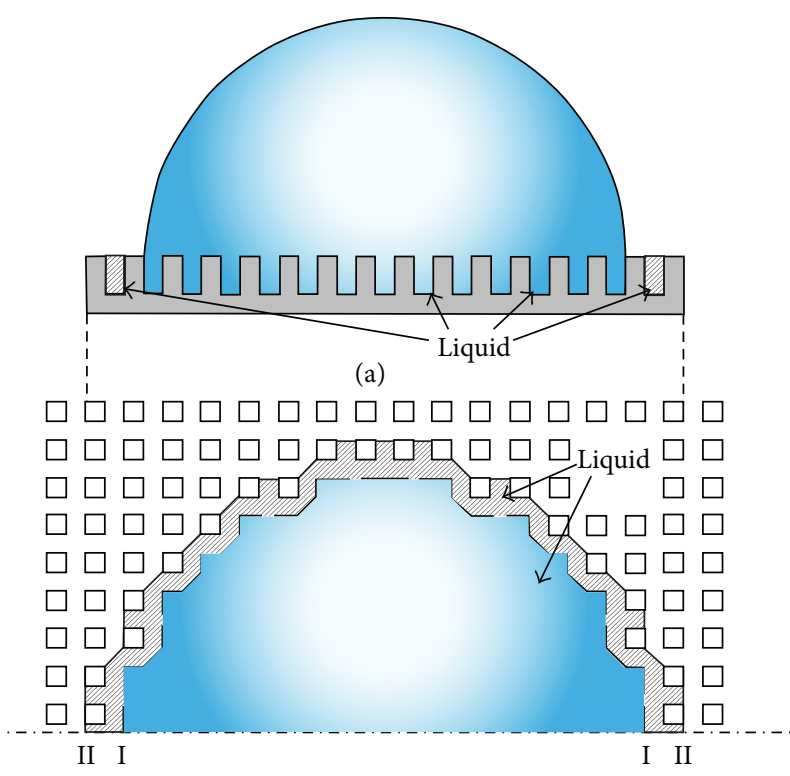

(b)

Figure 3: Cassie impregnating state.

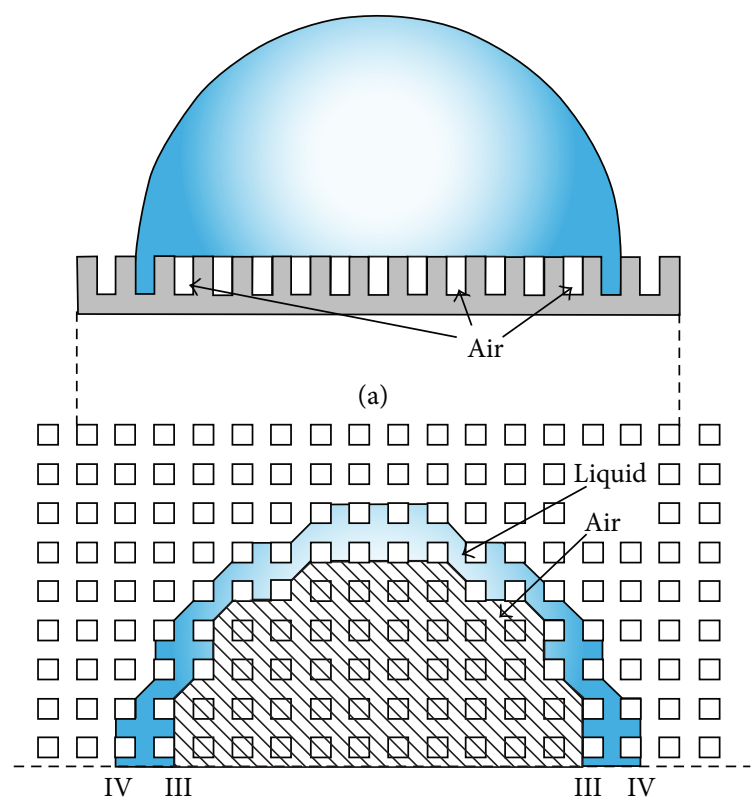

(b)

FIGURE 4: One-dimensional scenario of Wenzel state.

Wenzel state). Therefore, the effect of deformation of contact line was negligible.

When the inclination angle of solid surfaces reached a critical value, the droplet began to slide slowly, as shown in Figure 5. The volume of droplet here was $16 \mu \mathrm{L}$, and the area fraction was 0.17 . It was observed that the droplet sliding on the top of micropillars moved from one pillar to another pillar. The yellow arrow indicated the position of the droplet layer left behind on the micropillars, and the red arrow indicated particularly the droplet layer. The droplet layer began to evaporate quickly and then disappeared totally so that the droplet layer can easily be ignored.

To understand the process, a movement detail of the receding contact line as shown in Figure 6(a) was hypothesized, the wetting area at the top of the pillars remained constant, and the receding contact angle was increased gradually until the droplet sliding. It was shown in the experimental results that a thin droplet layer was left on the pillars as the droplet sliding on the pillars, as shown in Figure 6(b). Therefore, the wetting area at the top of the pillars still remained constant until the droplet completely separated from the pillars. Meanwhile, the receding contact line was constantly changing. In theoretical models, it was assumed that the receding contact angle kept constant as the droplet detached itself from the surface and the wetting area at the top of the first pillar became smaller, without considering droplet layers and the deformation of the receding contact line. Therefore, for the simulation results, it resulted in a smaller wetting area value [34].

To emphasize the deformation of contact lines and the remaining layer, we measured the sliding angle of droplets, comparing with theoretical models (see (3)), as shown in Figure 7 , respectively. When the area fraction was relatively large $(f>0.15)$, the experimental data were in good agreement with the theoretical model. However, the error increased with the decrease of the area fraction.

When the area fraction was large $(f>0.15)$, the receding contact angle was smaller than that of less area fraction. Obviously, the component interfacial tension along the direction of droplet motion would become smaller. With the decreasing of the area fraction, the component of surface tension $\gamma_{\mathrm{lv}}$ along the direction of droplet motion would increase, as shown in Figure 8(f) (the red dashed line indicated the air-liquid interface when the area fraction was small). In other words, with the increasing of the spacing between micropillars, the adhesion force would decrease. However, it caused the increasing resistance of the surface tension, resulting in the experimental results of sliding angle which were larger than the theoretical model deduced (3).

Experimental results show that as the inclination angle reached the critical value (sliding angle $\alpha$ ), the receding contact line of the droplet would move to the rear edge of micropillars. The droplet was unstable and the direction of $\gamma_{\mathrm{sv}}$ and $\gamma_{\mathrm{sl}}$ suddenly changed, as shown in Figures 8(c) and $8(\mathrm{~d})$. With the continuously increasing inclination angle, as shown in Figure 8(f), the receding contact line would quickly slide over the pillars and move to the next pillars with droplet layer left behind.

On the other hand, a droplet sliding on micropillared hydrophobic surfaces overcame both the work of adhesion and the corresponding energy barrier (EB). At present, the specific expression of EB was still unclear, but it was related to the area fraction, the volume of droplet, the sliding angle, and so forth. With the increasing spacing between micropillars, the area fraction decreased, and the EB increased. As shown in Figure 9, the reason that the micropillars affect the EB was presented in this work. There were different receding contact angles and sliding angle for the droplets due to the different area fractions. It was obvious that the spacing between 


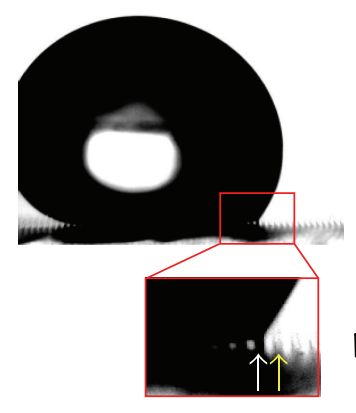

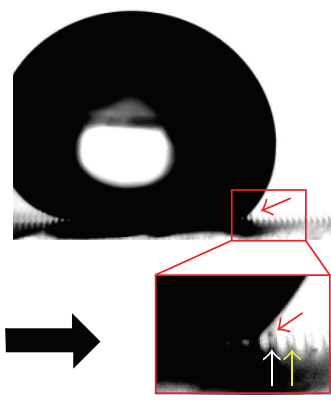

Droplet layer

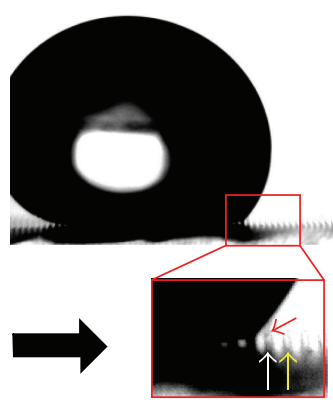

Evaporating

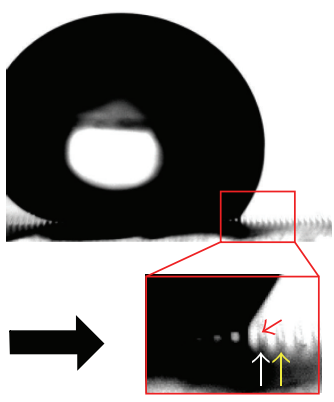

Evaporated

FIGURE 5: Droplet layer left behind a sliding droplet.

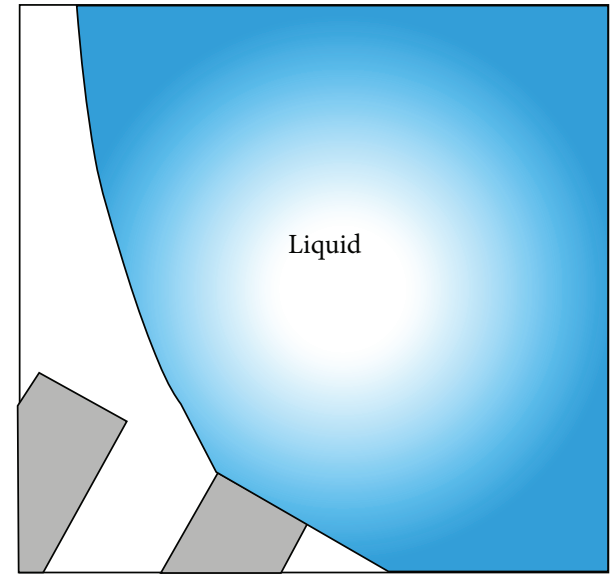

(a)

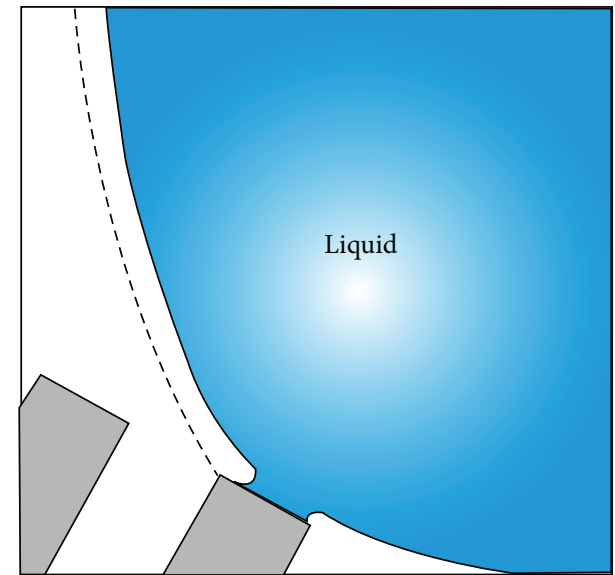

(b)

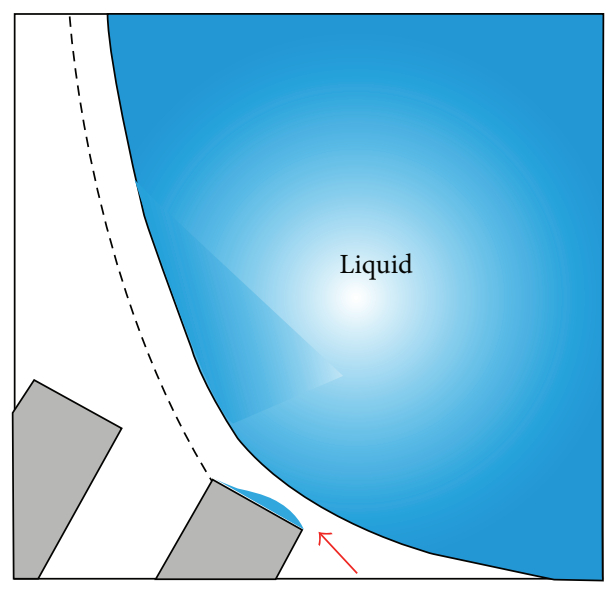

(c)

FIGURE 6: Detaching process of a droplet on micropillared surfaces.

micropillars in Figure 9(a) was less than in Figure 9(b) and the corresponding sliding angle was larger than in Figure 9(b). With the increasing inclination angle, the energy barrier of the droplet decreased. Obviously, the energy barrier which was overcome by droplets was that the energy barrier reached the sliding angle, known as the critical energy barrier (critical EB).
In Figure 9, a dotted line was drawn in front of the second pillar near the receding contact line, the droplet was separated into two parts, and their volumes were represented by $V_{1}$ and $V_{2}$, respectively. The droplet first slid on the topside of micropillars and then moved to the next pillars during the sliding process. During this process, $V_{2}$ of the droplet above the groove lost its support force from pillars, instead of the 

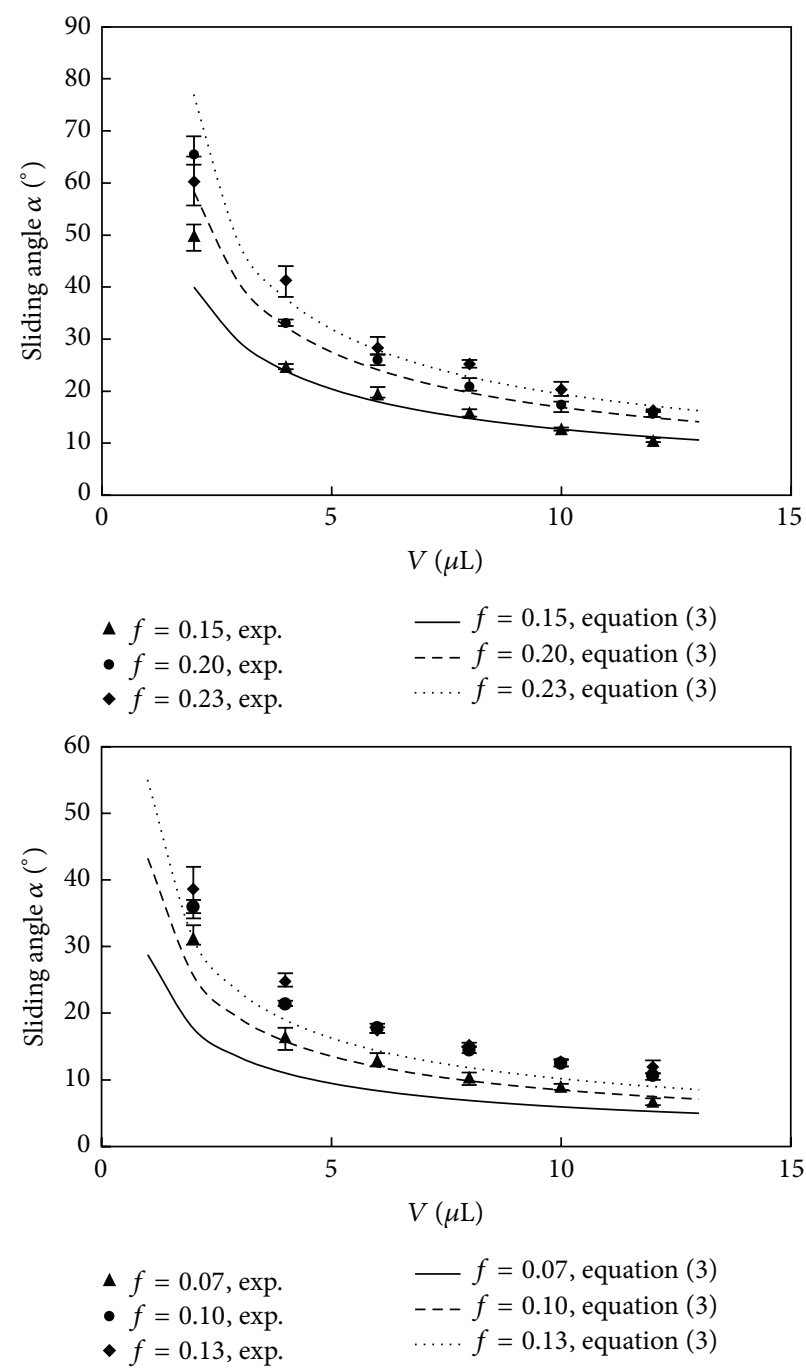

FIGURE 7: Comparison between experimental results and theoretical data.

drag force from $V_{1}$. At this moment, there was a certain resistance for $V_{2}$ to $V_{1}$. The larger the volume of $V_{2}$ was, the more the resistance existed. So the droplet would overcome the EB in the sliding process. It was obvious that with the increasing spacing between the pillars $V_{2}$ increased and $V_{1}$ decreased, and $V_{1}$ would decrease, so $V_{1} / V_{2}$ increased. As shown in Figure 9(b), in order to show the distinction, the two parts of the droplet were represented by $V_{1}^{\prime}$ and $V_{2}^{\prime}$, respectively. In Figure 9, $V_{2}^{\prime} / V_{1}^{\prime}>V_{2} / V_{1}$, and $V_{1}+V_{2}=$ $V_{1}^{\prime}+V_{2}^{\prime}$, so the critical EB of the droplet in Figure 9(b) was larger than that of Figure 9(a), which caused large error between the experimental results and the theoretical data.

\section{Summary and Conclusions}

Here, the dynamic behaviors of contact lines on micropillared surfaces were investigated. A series of experiments were performed to prove that droplet layer would be left behind after droplet detached from pillars. Therefore, the wetting area at the top of the pillars remained constant until the droplet completely separated from these pillars. The contact line changed into a ladder shape gradually and its shape was changing with time, so that the wetting area and the apparent contact angle were different at each instant. The theoretical values with experimental results were compared and analyzed by using force analysis of the receding contact line. It was found that the energy barrier was an important factor to construct a sliding angle model. Because of ignoring the role of the energy barrier, the theoretical model was not in accordance with experimental results, especially in small area fraction.

\section{Competing Interests}

The authors declare that there are no competing interests regarding the publication of this paper.

\section{Acknowledgments}

This study was funded by the National Natural Science Foundation of China under Grant nos. 51176123 and 51306123, Specialized Research Fund for the Doctoral Program of Higher 


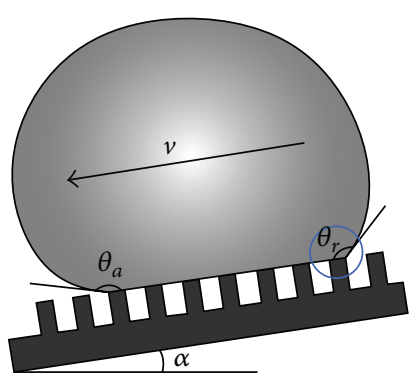

(a)

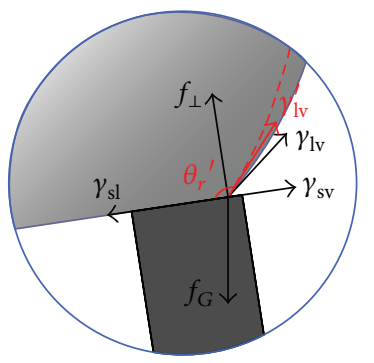

(f)

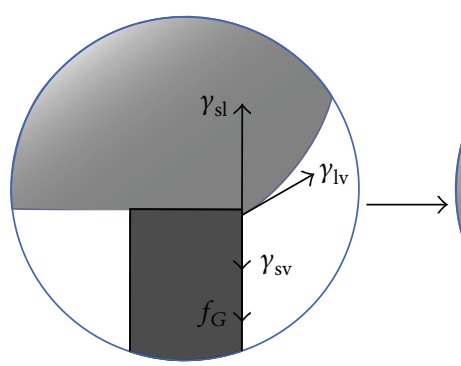

(b)

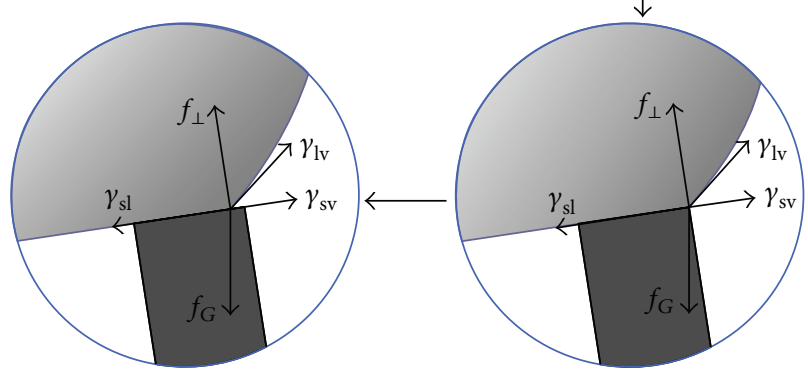

(e)

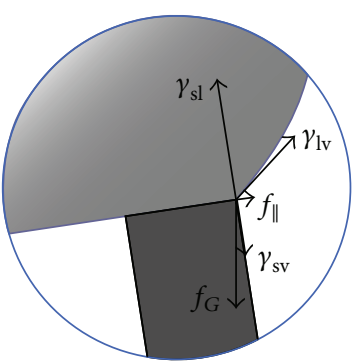

(c)

(d)

FIGURE 8: Force analysis of the receding contact line on micropillared surfaces.

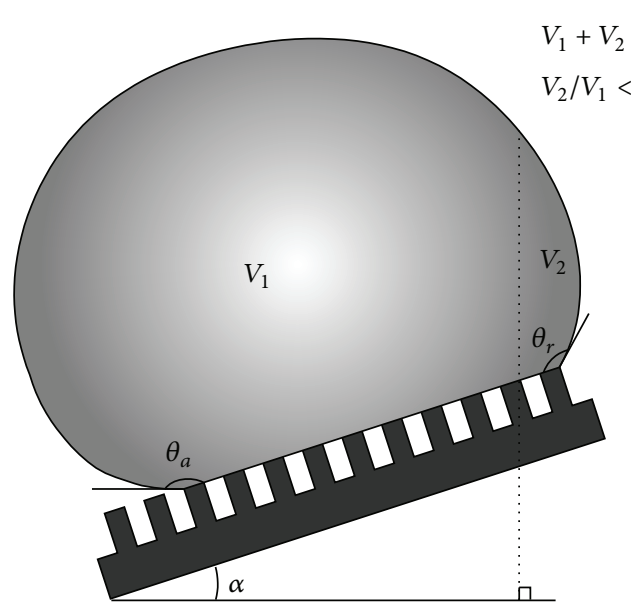

(a)

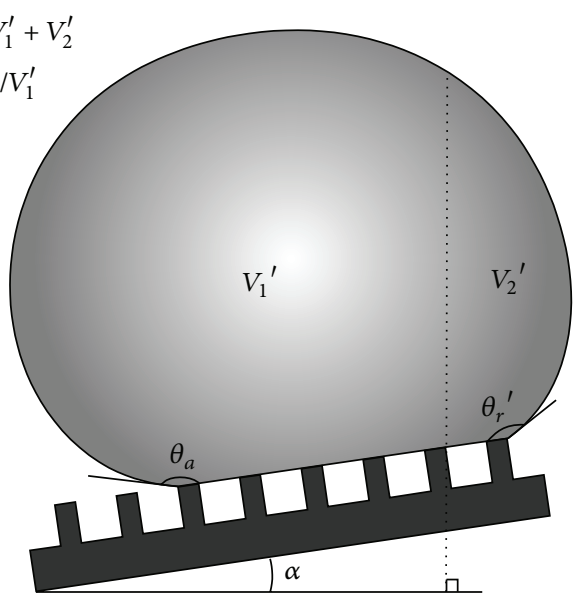

(b)

FIGURE 9: A droplet on inclined surfaces of different area fraction.

Education under Grant no. 20103120120006, and Shanghai Natural Science Fund under Grant no. 11ZR1424800.

\section{References}

[1] R. S. Voronov, D. V. Papavassiliou, and L. L. Lee, "Review of fluid slip over superhydrophobic surfaces and its dependence on the contact angle," Industrial and Engineering Chemistry Research, vol. 47, no. 8, pp. 2455-2477, 2008.

[2] J.-G. Fan, X.-J. Tang, and Y.-P. Zhao, "Water contact angles of vertically aligned Si nanorod arrays," Nanotechnology, vol. 15, no. 5, pp. 501-504, 2004.
[3] M. Kiuru and E. Alakoski, "Low sliding angles in hydrophobic and oleophobic coatings prepared with plasma discharge method," Materials Letters, vol. 58, no. 16, pp. 2213-2216, 2004.

[4] Y. Jiang, Z. Wang, X. Yu et al., "Self-assembled monolayers of dendron thiols for electrodeposition of gold nanostructures: toward fabrication of superhydrophobic/superhydrophilic surfaces and pH-responsive surfaces," Langmuir, vol. 21, no. 5, pp. 1986-1990, 2005.

[5] M. E. Abdelsalam, P. N. Bartlett, T. Kelf, and J. Baumberg, "Wetting of regularly structured gold surfaces," Langmuir, vol. 21, no. 5, pp. 1753-1757, 2005.

[6] L. Mishchenko, B. Hatton, V. Bahadur, J. A. Taylor, T. Krupenkin, and J. Aizenberg, "Design of ice-free nanostructured 
surfaces based on repulsion of impacting water droplets," ACS Nano, vol. 4, no. 12, pp. 7699-7707, 2010.

[7] N. J. Shirtcliffe, G. McHale, M. I. Newton, G. Chabrol, and C. C. Perry, "Dual-scale roughness produces unusually waterrepellent surfaces," Advanced Materials, vol. 16, no. 21, pp. 19291932, 2004.

[8] T. Sun, G. Wang, H. Liu, L. Feng, L. Jiang, and D. Zhu, "Control over the wettability of an aligned carbon nanotube film," Journal of the American Chemical Society, vol. 125, no. 49, pp. 1499614997, 2003.

[9] E. Martines, K. Seunarine, H. Morgan, N. Gadegaard, C. D. W. Wilkinson, and M. O. Riehle, "Superhydrophobicity and superhydrophilicity of regular nanopatterns," Nano Letters, vol. 5, no. 10, pp. 2097-2103, 2005.

[10] N. R. Tas, P. Mela, T. Kramer, J. W. Berenschot, and A. Van Den Berg, "Capillarity induced negative pressure of water plugs in nanochannels," Nano Letters, vol. 3, no. 11, pp. 1537-1540, 2003.

[11] C. W. Extrand, "Model for contact angles and hysteresis on rough and ultraphobic surfaces," Langmuir, vol. 18, no. 21, pp. 7991-7999, 2002.

[12] L. Gao and T. J. McCarthy, "Contact angle hysteresis explained," Langmuir, vol. 22, no. 14, pp. 6234-6237, 2006.

[13] L. Gao and T. J. McCarthy, "A perfectly hydrophobic surface $\left(\theta_{A} / \theta_{R}=180 \circ / 180 \circ\right)$," Journal of the American Chemical Society, vol. 128, no. 28, pp. 9052-9053, 2006.

[14] U. Thiele and E. Knobloch, "Driven drops on heterogeneous substrates: onset of sliding motion," Physical Review Letters, vol. 97, no. 20, Article ID 204501, 2006.

[15] R. Raj, R. Enright, Y. Zhu, S. Adera, and E. N. Wang, "Unified model for contact angle hysteresis on heterogeneous and superhydrophobic surfaces," Langmuir, vol. 28, no. 45, pp. 1577715788, 2012.

[16] J. H. Snoeijer and B. Andreotti, "Moving contact lines: scales, regimes, and dynamical transitions," Annual Review of Fluid Mechanics, vol. 45, pp. 269-292, 2013.

[17] Y. I. Frenkel, "On the behavior of liquid drops on a solid surface. 1. The sliding of drops on an inclined surface," Journal of Experimental and Theoretical Physics, vol. 18, p. 659, 1948.

[18] C. Dorrer and J. Rühe, "Advancing and receding motion of droplets on ultrahydrophobic post surfaces," Langmuir, vol. 22, no. 18, pp. 7652-7657, 2006.

[19] B. Widom, "Line tension and the shape of a sessile drop," Journal of Physical Chemistry, vol. 99, no. 9, pp. 2803-2806, 1995.

[20] P. Blecua, R. Lipowsky, and J. Kierfeld, "Line tension effects for liquid droplets on circular surface domains," Langmuir, vol. 22, no. 26, pp. 11041-11059, 2006.

[21] M. Brinkmann, J. Kierfeld, and R. Lipowsky, "A general stability criterion for droplets on structured substrates," Journal of Physics A: Mathematical and General, vol. 37, no. 48, pp. 1154711573, 2004.

[22] R. Lipowsky, P. Lenz, and P. S. Swain, "Wetting and dewetting of structured and imprinted surfaces," Colloids and Surfaces A: Physicochemical and Engineering Aspects, vol. 161, no. 1, pp. 322, 2000.

[23] P. S. Swain and R. Lipowsky, "Contact angles on heterogeneous surfaces: a new look at Lassie's and Werrzel's laws," Langmuir, vol. 14, no. 23, pp. 6772-6780, 1998.

[24] M. Reyssat and D. Quéré, "Contact angle hysteresis generated by strong dilute defects," The Journal of Physical Chemistry B, vol. 113, no. 12, pp. 3906-3909, 2009.
[25] R. Rosso and E. G. Virga, "Local stability for a general wetting functional," Journal of Physics A: Mathematical and General, vol. 37, no. 13, pp. 3989-4015, 2004.

[26] R. Rosso and E. G. Virga, "Sign of line tension in liquid bridge stability," Physical Review E, vol. 70, no. 3, Article ID 031603, 12 pages, 2004.

[27] J. F. Joanny and P. G. De Gennes, "A model for contact angle hysteresis," The Journal of Chemical Physics, vol. 81, no. 1, pp. 552-562, 1984.

[28] D. Öner and T. J. McCarthy, "Ultrahydrophobic surfaces. Effects of topography length scales on wettability," Langmuir, vol. 16, no. 20, pp. 7777-7782, 2000.

[29] S. Herminghaus, "Roughness-induced non-wetting," EPL (Europhysics Letters), vol. 52, no. 2, p. 165, 2007.

[30] A. T. Paxson and K. K. Varanasi, "Self-similarity of contact line depinning from textured surfaces," Nature Communications, vol. 4, article 1492, 2013.

[31] C. G. L. Furmidge, "Studies at phase interfaces. I. The sliding of liquid drops on solid surfaces and a theory for spray retention," Journal of Colloid Science, vol. 17, no. 4, pp. 309-324, 1962.

[32] E. Wolfram, "Comment on the paper "study of wettability of polymers by sliding of water drops" by Koji Kawasaki," Journal of Colloid Science, vol. 16, no. 2, pp. 195-197, 1961.

[33] M. Miwa, A. Nakajima, A. Fujishima, K. Hashimoto, and T. Watanabe, "Effects of the surface roughness on sliding angles of water droplets on superhydrophobic surfaces," Langmuir, vol. 16, no. 13, pp. 5754-5760, 2000.

[34] C. Lv, C. Yang, P. Hao, F. He, and Q. Zheng, "Sliding of water droplets on microstructured hydrophobic surfaces," Langmuir, vol. 26, no. 11, pp. 8704-8708, 2010.

[35] E. Bormashenko, R. Pogreb, G. Whyman, and M. Erlich, "Cassie-Wenzel wetting transition in vibrating drops deposited on rough surfaces: is the dynamic Cassie-Wenzel wetting transition a 2D or 1D affair?" Langmuir, vol. 23, no. 12, pp. 65016503, 2007.

[36] E. Bormashenko, A. Musin, G. Whyman, and M. Zinigrad, "Wetting transitions and depinning of the triple line," Langmuir, vol. 28, no. 7, pp. 3460-3464, 2012. 

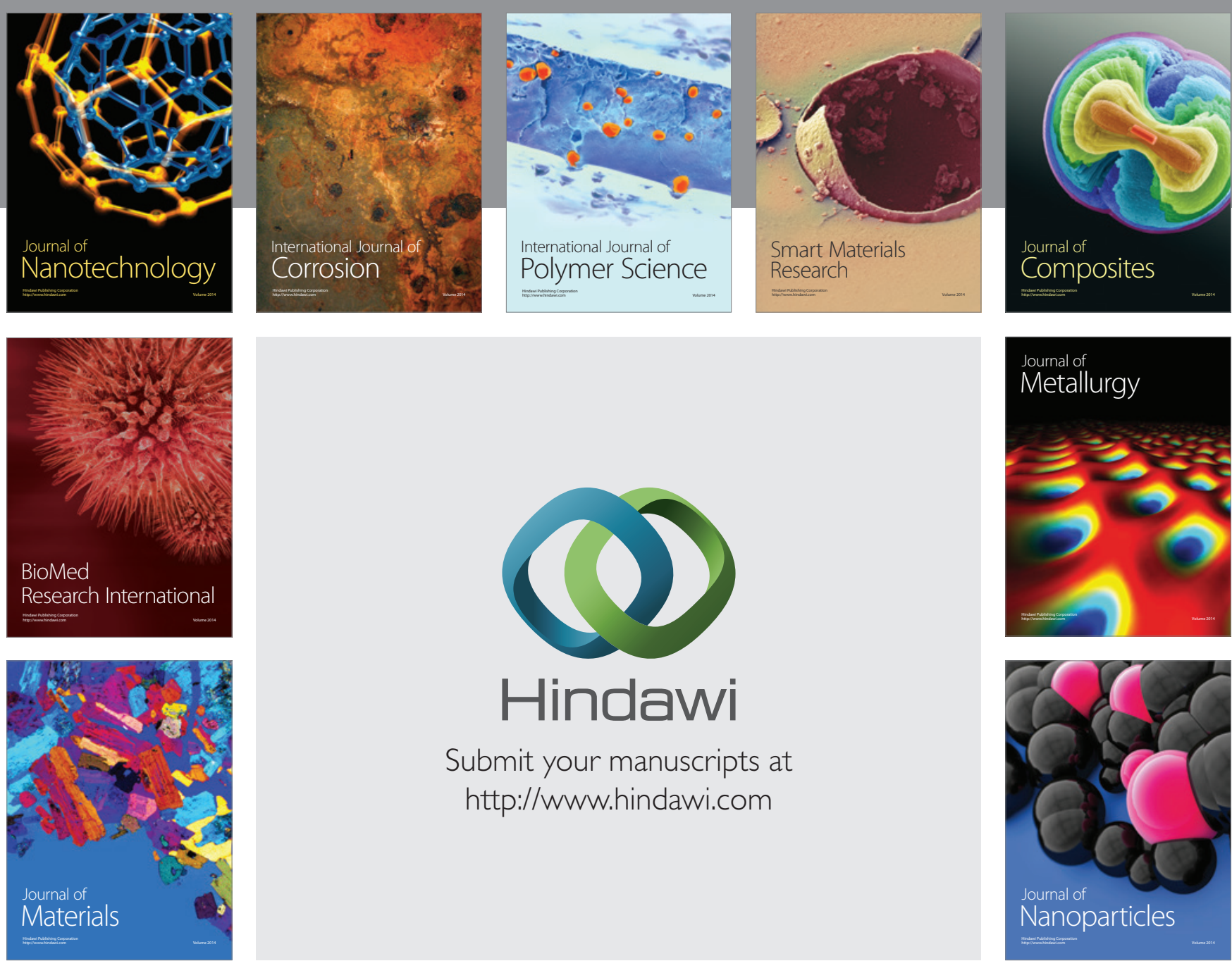

\section{Hindawi}

Submit your manuscripts at

http://www.hindawi.com

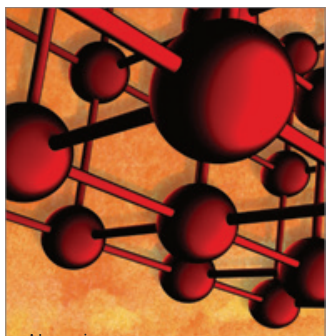

Materials Science and Engineering
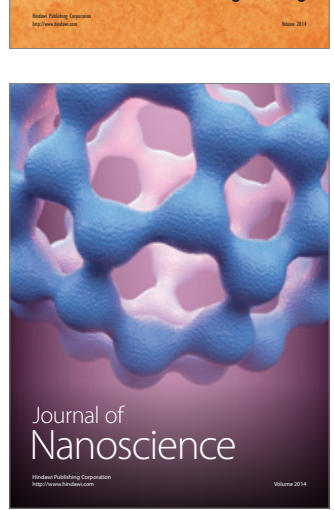
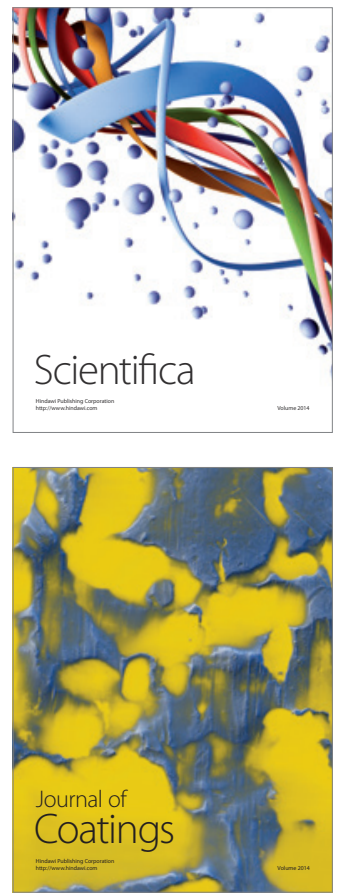
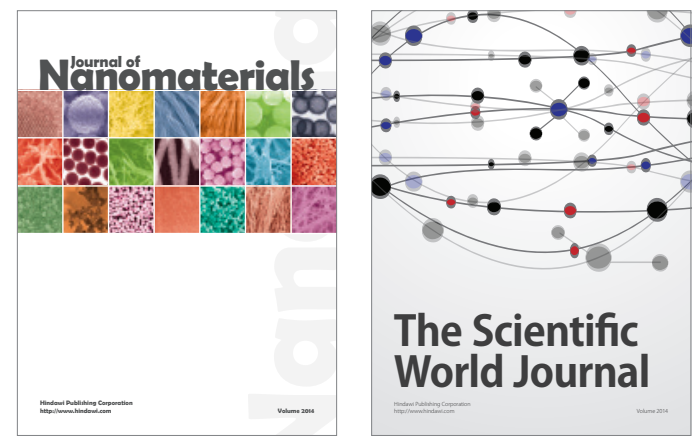

The Scientific World Journal
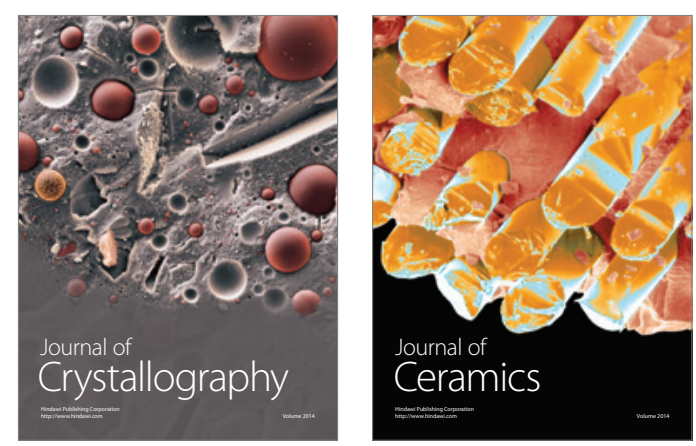
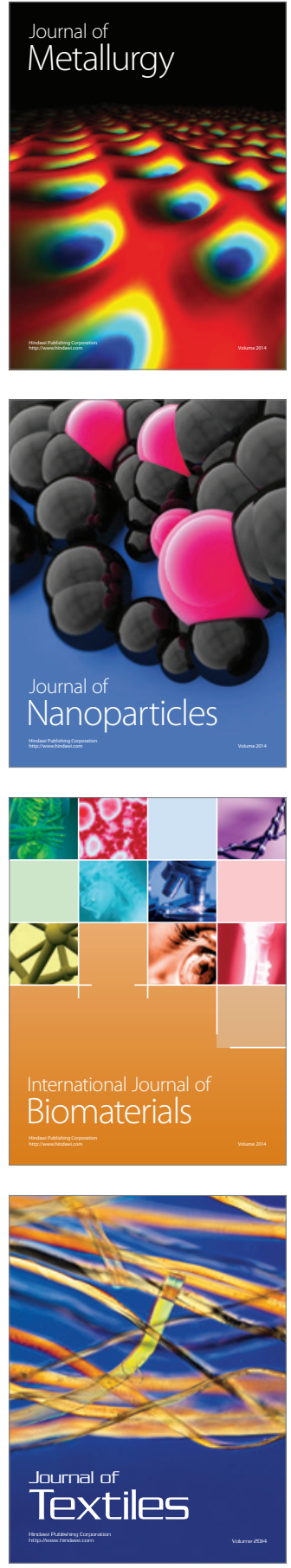